University of Nebraska - Lincoln

DigitalCommons@University of Nebraska - Lincoln

Publications, Agencies and Staff of the U.S.

Department of Commerce

U.S. Department of Commerce

2008

Spatial methods for plot-based sampling of wildlife populations

Jay M. Ver Hoef

Alaska Department of Fish and Game

Follow this and additional works at: https://digitalcommons.unl.edu/usdeptcommercepub

Part of the Environmental Sciences Commons

Ver Hoef, Jay M., "Spatial methods for plot-based sampling of wildlife populations" (2008). Publications, Agencies and Staff of the U.S. Department of Commerce. 183.

https://digitalcommons.unl.edu/usdeptcommercepub/183

This Article is brought to you for free and open access by the U.S. Department of Commerce at DigitalCommons@University of Nebraska - Lincoln. It has been accepted for inclusion in Publications, Agencies and Staff of the U.S. Department of Commerce by an authorized administrator of DigitalCommons@University of Nebraska - Lincoln. 


\title{
Spatial methods for plot-based sampling of wildlife populations
}

\author{
Jay M. Ver Hoef
}

Received: 1 August 2005 / Revised: 1 May 2006 / Published online: 19 September 2007

(C) Springer Science+Business Media, LLC 2007

\begin{abstract}
Classical sampling methods can be used to estimate the mean of a finite or infinite population. Block kriging also estimates the mean, but of an infinite population in a continuous spatial domain. In this paper, I consider a finite population version of block kriging (FPBK) for plot-based sampling. The data are assumed to come from a spatial stochastic process. Minimizing mean-squared-prediction errors yields best linear unbiased predictions that are a finite population version of block kriging. FPBK has versions comparable to simple random sampling and stratified sampling, and includes the general linear model. This method has been tested for several years for moose surveys in Alaska, and an example is given where results are compared to stratified random sampling. In general, assuming a spatial model gives three main advantages over classical sampling: (1) FPBK is usually more precise than simple or stratified random sampling, (2) FPBK allows small area estimation, and (3) FPBK allows nonrandom sampling designs.
\end{abstract}

Keywords Geostatistics · Variogram - Block kriging · Finite population - BLUP . Small area

\section{Introduction}

Monitoring ecological populations is an important goal for both academic research and management of natural resources. Successful management of moose populations in Alaska depends on obtaining estimates of moose abundance at regular intervals throughout the state. The Alaska Department of Fish and Game developed aerial survey methods to estimate and monitor moose populations (Gasaway et al. 1986). The methods of Gasaway et al. (1986) use stratified random sampling and are based on classical sampling principles that rely on

J. M. Ver Hoef

Alaska Department of Fish and Game, 1300 College Road, Fairbanks AK 99701, USA

J. M. Ver Hoef $(\bowtie)$

National Marine Mammal Laboratory, 7600 Sand Point Way NE, Bldg 4, Seattle WA 98115-6349, USA

e-mail: jay.verhoef@noaa.gov 
design-based inference, which are very robust. Very few assumptions are required because the distribution for inference comes from the sample design, which is known and under our control. In this paper we will be interested in estimating (predicting) the mean or total number of moose from a fixed geographic area. For design-based methods, sample plots are chosen at random, moose are counted in these plots, and inference is derived from the inclusion probability for sample units (i.e., Horwitz-Thompson estimation). For moose surveys there are a finite number of sample units and so finite population methods are used.

There are some problems with design-based methods. Because few assumptions are required, they may lack power in cases where further assumptions are justified. This appears to be especially true in the case of "small area" estimation, which refers to making an estimate on a smaller geographic area within the overall study area. There may be few or no samples within that small area, so that design-based estimation may not be possible or variances become exceedingly large. An alternative is to assume that the data were generated by a stochastic process and use model-based approaches (see e.g., Fay and Harriot 1979; Ghosh and Meeden 1986; Prasad and Rao 1990).

The basic problem considered in this paper is the estimation of some function of the sample units, call it $\tau(\mathbf{z})$, where $\mathbf{z}$ is a vector of the realized values of a spatial stochastic process for all the sample units of a finite population. The function $\tau(\mathbf{z})$ could be the population mean, population total, or the mean or total of a subset of sample units that have few or no observed samples. The goal is to use a predictor based on the set of observed samples $\widehat{\tau}\left(\mathbf{z}_{s}\right)$, where $\mathbf{z}_{S}$ is a vector of observed values for sampled units (see e.g., Bolfarine and Zacks 1992, p. 6). Geostatistical models and methods are used (for a review, see Cressie 1993). Geostatistics has been developed for point samples. Because points are infinitesimally small, an infinite population is assumed. The average value over some area can be predicted using methods such as block kriging, which uses aggregation. Thus it appears that this is closely related to small area estimation, but where samples come from point locations rather than a finite set of sample units. In this paper, I consider the case where we have a finite collection of plots and we assume that the data were produced by a spatial stochastic process. It appears this has not been considered in detail. I develop a finite population version of block kriging (FPBK) which has been successfully used for estimating and monitoring moose abundance in Alaska, the Yukon, and the Northwest Territories.

\subsection{Quick review of universal block kriging}

Kriging is a spatial prediction method that is formulated by minimizing the mean-squaredprediction errors (MSPE), also known as the prediction variance. This treatment follows Cressie (1993, p. 151). Kriging can be formulated by using variograms or covariance. Here, we show the covariance results. Suppose the data follow some linear model,

$$
\mathbf{z}=\boldsymbol{\mu}+\boldsymbol{\delta},
$$

where $\boldsymbol{\mu}=\mathbf{X} \boldsymbol{\beta}$ and $\mathbf{X}$ has dimensions $n \times p$. Assume that the spatial random variable $Z(\mathbf{s})$ is defined at each location $\mathbf{s}$ in some region $\mathcal{D} \subset \mathcal{R}^{d}$. Define second-order stationarity for the random errors $\delta$ as follows: $E[\delta(\mathbf{s})]=0$ so that $E[Z(\mathbf{s})]=\mu(\mathbf{s})=\mathbf{x}^{\prime}(\mathbf{s}) \boldsymbol{\beta}$ for all $\mathbf{s} \in \mathcal{D}$, and that the covariance,

$$
C(\mathbf{h}) \equiv \operatorname{cov}[\delta(\mathbf{s}), \delta(\mathbf{s}+\mathbf{h})],
$$

exists and depends only on $\mathbf{h}$. For universal block kriging, define

$$
Z(B) \equiv \int_{B} Z(\mathbf{s}) d \mathbf{s} /|B|,
$$


and

$$
\mu(B) \equiv \int_{B} \mu(\mathbf{s}) d \mathbf{s} /|B|,
$$

for some area $B \subset \mathcal{D}$ where $|B|$ is the area (volume) of $B$, assuming that the integrals exist for the process $\{Z(\mathbf{s})\}$ (see Cressie 1993, p. 106). $Z(B)$ is a random variable for the average value within the block $B$, and $\mu(B)$ is the expected value within the block. Data are collected at $n$ locations, and assume the data are a realization of the random vector $\mathbf{z} \equiv\left[Z\left(\mathbf{s}_{1}\right), Z\left(\mathbf{s}_{2}\right), \ldots, Z\left(\mathbf{s}_{n}\right)\right]$. Let $\mathbf{a}^{\prime} \mathbf{z}$ be a linear predictor for the random variable $Z(B)$, subject to the unbiasedness constraint $E\left(\mathbf{a}^{\prime} \mathbf{z}\right)=E[Z(B)]$. Then universal block kriging uses (2) to minimize the MSPE; that is, find a $\lambda$ such that

$$
E\left[\mathbf{a}^{\prime} \mathbf{z}-Z(B)\right]^{2}-E\left[\lambda^{\prime} \mathbf{z}-Z(B)\right]^{2} \geq 0
$$

for all a such that $\mathbf{a}^{\prime} \mathbf{z}$ is unbiased. Minimizing $E\left[\lambda^{\prime} \mathbf{z}-Z(B)\right]^{2}$ in (4) in terms of covariances yields the set of equations,

$$
\left(\begin{array}{cc}
\Sigma & \mathbf{X} \\
\mathbf{X}^{\prime} & \mathbf{0}
\end{array}\right)\left(\begin{array}{c}
\lambda \\
\mathbf{m}
\end{array}\right)=\left(\begin{array}{l}
\mathbf{c}_{B} \\
\mathbf{x}_{B}
\end{array}\right),
$$

where $\mathbf{c}_{B}=\left[c_{1}(B), c_{2}(B), \ldots, c_{n}(B)\right]^{\prime}$ with $c_{i}(B) \equiv \int_{B} C\left(\mathbf{s}-\mathbf{s}_{i}\right) d \mathbf{s} /|B|$ for $i=1,2, \ldots$, $n, \mathbf{x}_{B}=\left[x_{1}(B), x_{2}(B), \ldots, x_{p}(B)\right]^{\prime}$ with $x_{j}(B) \equiv \int_{B} x_{j}(\mathbf{s}) d \mathbf{s} /|B|$ for $j=1,2, \ldots, p$, and $\mathbf{m}$ are the Lagrange multipliers due to the unbiasedness constraint. The solution of (5) for $\lambda$ and $\mathbf{m}$ yields the block BLUP $\widehat{Z}(B)=\lambda^{\prime} \mathbf{z}$, which can be written as

$$
\widehat{Z}(B)=\mathbf{c}_{B}^{\prime} \Sigma^{-1}(\mathbf{z}-\widehat{\boldsymbol{\mu}})+\widehat{\mu}_{B},
$$

where $\widehat{\boldsymbol{\mu}} \equiv \mathbf{X} \widehat{\boldsymbol{\beta}}_{G L S}$ and $\widehat{\mu}_{B} \equiv \mathbf{x}_{B}^{\prime} \widehat{\boldsymbol{\beta}}_{G L S}$ with $\widehat{\boldsymbol{\beta}}_{G L S}=\left(\mathbf{X}^{\prime} \Sigma^{-1} \mathbf{X}\right)^{-1} \mathbf{X}^{\prime} \Sigma^{-1} \mathbf{z}$. The block kriging variance is given by

$$
E\left[\lambda^{\prime} \mathbf{z}-Z(B)\right]^{2}=\sigma_{B, B}^{2}-\mathbf{c}_{B}^{\prime} \Sigma^{-1} \mathbf{c}_{B}+\mathbf{d}_{B}^{\prime}\left(\mathbf{X}^{\prime} \Sigma^{-1} \mathbf{X}\right)^{-1} \mathbf{d}_{B},
$$

where $\sigma_{B, B}^{2}$ is $\int_{B} \int_{B} C(\mathbf{s}-\mathbf{u}) d \mathbf{s} d \mathbf{u} /|B|^{2}$ and $\mathbf{d}_{B}=\left(\mathbf{x}_{B}-\mathbf{X}^{\prime} \Sigma^{-1} \mathbf{c}_{B}\right)$.

\section{Block prediction for finite populations}

One objective of finite population sampling is to estimate the average or total of the values that are actually realized, rather than the mean of some superpopulation from which the data were drawn. The equivalent objective is prediction, not estimation, for spatial processes in model-based approaches. That is, the goal is to predict a function of the actual values that occurred, not estimate unobservable parameters of a model (see Cressie 1993, pp. 13-16 for more details). To formulate this more clearly, suppose that $\mathbf{z}$ is a vector of random variables on a finite spatial lattice. The spatial lattice $D$ is a set that can be indexed, each location denoted by $i, i=1,2, \ldots, N$. The random variable $Z_{i}$ is located at the $i$ th site in the lattice. Let $\boldsymbol{\tau}(\mathbf{z})=\mathbf{B}^{\prime} \mathbf{z}$ be a vector of random variables to be predicted, where $\mathbf{B}$ is the $N \times k$ matrix $\left[\mathbf{b}_{1}\left|\mathbf{b}_{2}\right| \cdots \mid \mathbf{b}_{k}\right]$. For example, $\mathbf{b}_{j}=(1 / N)(1,1, \ldots, 1)^{\prime}$ would be the average of the realized values of $\mathbf{z}$ in $D$. Notice that here $\mathbf{b}^{\prime} \mathbf{z}$ acts as the finite version of $Z(B)$ in (3). Other possibilities are $\mathbf{b}_{j}=(1,1, \ldots, 1)^{\prime}$, which is the total of the realized values of $\mathbf{z}$ in $D$, and $\mathbf{b}_{j}=(0,0, \ldots, 0,1,1, \ldots, 1,0, \ldots, 0,0)^{\prime}$ which is the total of the realized values of $\mathbf{z}$ for some subregion (small area) in $D$. Data are collected from a subset of $D$, call it the 
$n \times 1$ vector $\mathbf{z}_{S}$, and let the unsampled locations be denoted by the $(N-n) \times 1$ vector $\mathbf{z}_{u}$, and write $\mathbf{z}=\left(\mathbf{z}_{s}^{\prime}, \mathbf{z}_{u}^{\prime}\right)^{\prime}$. We want some linear combination of the data, call it $\widehat{\boldsymbol{\tau}}\left(\mathbf{z}_{s}\right)=\mathbf{A}^{\prime} \mathbf{z}_{s}$, in order to predict $\mathbf{B}^{\prime} \mathbf{z}$.

Definition 1 Mean-Squared Prediction Error (MSPE) Matrix

Let the MSPE matrix for any particular $\mathbf{A}$ be,

$$
\mathbf{M}_{A}=E\left(\mathbf{A}^{\prime} \mathbf{z}_{s}-\mathbf{B}^{\prime} \mathbf{z}\right)\left(\mathbf{A}^{\prime} \mathbf{z}_{s}-\mathbf{B}^{\prime} \mathbf{z}\right)^{\prime} .
$$

Definition 2 Best Linear Unbiased Predictor (BLUP)

The predictor $\mathbf{\Lambda}^{\prime} \mathbf{z}$ is BLUP if,

(1) $E\left(\Lambda^{\prime} \mathbf{z}_{S}\right)=E\left(\mathbf{B}^{\prime} \mathbf{z}\right)$, and

(2) $\mathbf{M}_{A}-\mathbf{M}_{\Lambda}$ is non-negative definite for every $\mathbf{A} \neq \boldsymbol{\Lambda}$.

For the rest of this paper, assume that $\mathbf{z}$ follows the linear model, $\mathbf{z}=\mathbf{X} \boldsymbol{\beta}+\boldsymbol{\delta}$, or

$$
\left(\begin{array}{c}
\mathbf{z}_{s} \\
\mathbf{z}_{u}
\end{array}\right)=\left(\begin{array}{l}
\mathbf{X}_{s} \\
\mathbf{X}_{u}
\end{array}\right) \boldsymbol{\beta}+\left(\begin{array}{l}
\boldsymbol{\delta}_{s} \\
\boldsymbol{\delta}_{u}
\end{array}\right),
$$

where $\mathbf{X}$ is a matrix of fixed effects, $\boldsymbol{\beta}$ is a parameter vector, $E(\boldsymbol{\delta})=\mathbf{0}$, and

$$
\operatorname{var}(\boldsymbol{\delta})=\left(\begin{array}{cc}
\Sigma_{s s} & \Sigma_{s u} \\
\Sigma_{u s} & \Sigma_{u u}
\end{array}\right)
$$

To find the BLUP, we need to establish the uniform unbiasedness conditions for the predictor and then find the $\boldsymbol{\Lambda}$ that "minimizes" the MSPE matrix.

\subsection{Uniform unbiasedness conditions}

We need to consider all $\mathbf{A}$ such that $E\left(\mathbf{A}^{\prime} \mathbf{z}_{s}\right)=E\left(\mathbf{B}^{\prime} \mathbf{z}\right)$ for all $\boldsymbol{\beta}$ in the linear model (9). Taking expectations, we see that $\mathbf{A}^{\prime} \mathbf{X}_{s} \boldsymbol{\beta}=\mathbf{B}^{\prime} \mathbf{X} \boldsymbol{\beta}$ for every $\boldsymbol{\beta}$, so that implies $\mathbf{A}^{\prime} \mathbf{X}_{s}=\mathbf{B}^{\prime} \mathbf{X}$, or

$$
\mathbf{A}^{\prime} \mathbf{X}_{s}=\mathbf{B}_{s}^{\prime} \mathbf{X}_{s}+\mathbf{B}_{u}^{\prime} \mathbf{X}_{u}
$$

where $\mathbf{B}^{\prime}=\left[\mathbf{B}_{s}^{\prime} \mid \mathbf{B}_{u}^{\prime}\right]$.

2.2 Prediction for finite populations

Similar to Eq. 4, the BLUP is found by finding $\boldsymbol{\Lambda}$ such that

$$
E\left(\mathbf{A}^{\prime} \mathbf{z}_{s}-\mathbf{B}^{\prime} \mathbf{z}\right)\left(\mathbf{A}^{\prime} \mathbf{z}_{s}-\mathbf{B}^{\prime} \mathbf{z}\right)-E\left(\mathbf{\Lambda}^{\prime} \mathbf{z}_{s}-\mathbf{B}^{\prime} \mathbf{z}\right)\left(\mathbf{\Lambda}^{\prime} \mathbf{z}_{s}-\mathbf{B}^{\prime} \mathbf{z}\right)
$$

is non-negative definite for all $\mathbf{A}$ such that $\mathbf{A}^{\prime} \mathbf{z}_{S}$ is unbiased. By minimizing the MSPE matrix, we obtain the prediction equations,

$$
\left(\begin{array}{ll}
\Sigma_{s s} & \mathbf{X}_{s} \\
\mathbf{X}_{s}^{\prime} & \mathbf{0}
\end{array}\right)\left(\begin{array}{l}
\mathbf{\Lambda} \\
\mathbf{M}
\end{array}\right)=\left(\begin{array}{cc}
\Sigma_{s s} & \Sigma_{s u} \\
\mathbf{X}_{s}^{\prime} & \mathbf{X}_{u}^{\prime}
\end{array}\right)\left(\begin{array}{l}
\mathbf{B}_{s} \\
\mathbf{B}_{u}
\end{array}\right),
$$

which can be compared to Eq. 5. When (12) are solved for $\boldsymbol{\Lambda}$, the FPBK predictor is,

$$
\boldsymbol{\tau}\left(\mathbf{B}^{\prime} \mathbf{z}\right)=\Lambda^{\prime} \mathbf{z}_{s}=\mathbf{B}_{s}^{\prime} \mathbf{z}_{s}+\mathbf{B}_{u}^{\prime} \widehat{\mathbf{z}}_{u},
$$

where,

$$
\widehat{\mathbf{z}}_{u}=\Sigma_{u s} \Sigma_{s s}^{-1}\left(\mathbf{z}_{s}-\widehat{\boldsymbol{\mu}}_{s}\right)+\widehat{\boldsymbol{\mu}}_{u},
$$


$\widehat{\boldsymbol{\mu}}_{u}=\mathbf{X}_{\mathbf{u}} \widehat{\boldsymbol{\beta}}_{G L S}$ and $\widehat{\boldsymbol{\mu}}_{s}=\mathbf{X}_{\mathbf{s}} \widehat{\boldsymbol{\beta}}_{G L S}$ with $\widehat{\boldsymbol{\beta}}_{G L S}=\left(\mathbf{X}_{s}^{\prime} \Sigma_{s s}^{-1} \mathbf{X}_{\mathbf{s}}\right)^{-1} \mathbf{X}_{\mathbf{s}}^{\prime} \Sigma_{s s}^{-1} \mathbf{z}_{s}$. The predictor (14) can be compared to (6). The FPBK predictor (13) is now seen as multiplying the observed sample values times their corresponding coefficients from $\mathbf{B}_{s}$, and then using universal block kriging to predict all other unsampled units, and these predictions are multiplied by their corresponding coefficients in $\mathbf{B}_{u}$.

\subsection{Prediction variance (MSPE)}

Substituting the solution for $\boldsymbol{\Lambda}$ for $\mathbf{A}$ in (8), we obtain the MSPE $\mathbf{M}_{\boldsymbol{\Lambda}}$ (also called prediction variance) of FPBK,

$$
\mathbf{M}_{\Lambda}=\mathbf{B}^{\prime} \Sigma \mathbf{B}-\mathbf{C}^{\prime} \Sigma_{s s}^{-1} \mathbf{C}+\mathbf{D}^{\prime} \mathbf{V D},
$$

where

$$
\begin{aligned}
& \mathbf{C}=\Sigma_{s s} \mathbf{B}_{s}+\Sigma_{s u} \mathbf{B}_{u}, \\
& \mathbf{D}=\mathbf{X}^{\prime} \mathbf{B}-\mathbf{X}_{\mathbf{s}}^{\prime} \Sigma_{s s}^{-1} \mathbf{C}, \text { and } \\
& \mathbf{V}=\operatorname{var}\left(\widehat{\boldsymbol{\beta}}_{G L S}\right)=\left(\mathbf{X}_{s}^{\prime} \Sigma_{s s}^{-1} \mathbf{X}_{s}\right)^{-1} .
\end{aligned}
$$

Equation 15 can be compared to (7). In (15), the quantity $\mathbf{B}^{\prime} \Sigma \mathbf{B}$ is the variance of $\mathbf{B}^{\prime} \mathbf{z}$, and assuming $\boldsymbol{\beta}$ is known, the prediction variance of $\mathbf{B}^{\prime} \mathbf{z}$ is $\mathbf{B}^{\prime} \Sigma \mathbf{B}-\mathbf{C}^{\prime} \Sigma_{s s}^{-1} \mathbf{C}$. The additional term $\mathbf{D}^{\prime} \mathbf{V D}$ arises because we are estimating $\boldsymbol{\beta}$, where $\mathbf{D}$ is, in some sense, the distance between predicted points in the design matrix $\mathbf{B}^{\prime} \mathbf{X}$ and that of the observed design matrix $\mathbf{C}^{\prime} \Sigma_{s s}^{-1} \mathbf{X}_{s}$. Equation 15 can be simplified for computing purposes,

$$
\mathbf{M}_{\Lambda}=\mathbf{B}_{u}^{\prime}\left(\Sigma_{u u}-\Sigma_{u s} \Sigma_{s s}^{-1} \Sigma_{s u}+\mathbf{W}^{\prime} \mathbf{V W}\right) \mathbf{B}_{u},
$$

where $\mathbf{W}=\mathbf{X}_{u}^{\prime}-\mathbf{X}_{\mathbf{s}}^{\prime} \Sigma_{s s}^{-1} \Sigma_{s u}$. If $\mathbf{B}$ has more than one column, the prediction variances of each $\mathbf{b}_{j}$ are contained as diagonal elements of $\mathbf{M}_{\boldsymbol{\Lambda}}$ and prediction covariances between $\mathbf{b}_{j}$ and $\mathbf{b}_{j^{\prime}}$ are contained as the off-diagonal elements of $\mathbf{M}_{\boldsymbol{\Lambda}}$.

\subsection{Connections to sampling theory}

Suppose we are interested in predicting the mean over a lattice of $N$ sites. Then $\mathbf{B}=\mathbf{b}=$ $(1 / N)(1,1, \ldots, 1)^{\prime}$. Assume no spatial autocorrelation; i.e., let $\boldsymbol{\Sigma}_{s s}=\sigma^{2} \mathbf{I}_{n}$ where $\mathbf{I}_{n}$ is the $n \times n$ identity matrix, $\boldsymbol{\Sigma}_{u u}=\sigma^{2} \mathbf{I}_{N-n}$ where $\mathbf{I}_{N-n}$ is the $(N-n) \times(N-n)$ identity matrix, $\boldsymbol{\Sigma}_{s u}=\boldsymbol{\Sigma}_{u s}^{\prime}=\mathbf{0}$, and $\mathbf{X}=\mathbf{1}_{N}$, where $\mathbf{1}_{N}$ is a vector of $N$ ones. Then from ( 13) $\boldsymbol{\Lambda}=\boldsymbol{\lambda}=(1 / n) \mathbf{1}_{n}$ and the predictor is the sample mean

$$
\lambda^{\prime} \mathbf{z}_{s}=\bar{z}
$$

Likewise, from (15) the

$$
\operatorname{MSPE}=\left(\sigma^{2} / n\right)(1-f),
$$

where $f=(n / N)$ is the sampling fraction and $1-f$ is the finite population correction factor. Of course, Eq. 16 is the same estimator of the mean that is used in simple random sampling (e.g., Thompson 1992, p. 13) and Eq. 17 is the variance of the mean estimator used in simple random sampling (e.g., Thompson 1992, p. 15). 
Next, consider stratified sampling. Allow each stratum to be a separate random process, independent from each other, each with its own mean and variance. These are model-based assumptions that are equivalent to stratified random sampling (SRS),

$$
\mathbf{X}=\left(\begin{array}{ll}
\mathbf{1}_{n_{1}} & \mathbf{0} \\
\mathbf{0} & \mathbf{1}_{n_{2}} \\
\mathbf{1}_{N_{1}-n_{1}} & \mathbf{0} \\
\mathbf{0} & \mathbf{1}_{N_{2}-n_{2}}
\end{array}\right)
$$

and

$$
\begin{aligned}
& \boldsymbol{\Sigma}_{s s}=\left(\begin{array}{ll}
\sigma_{1}^{2} \mathbf{I}_{n_{1}} & \mathbf{0} \\
\mathbf{0} & \sigma_{2}^{2} \mathbf{I}_{n_{2}}
\end{array}\right), \\
& \boldsymbol{\Sigma}_{u u}=\left(\begin{array}{ll}
\sigma_{1}^{2} \mathbf{I}_{N_{1}-n_{1}} & \mathbf{0} \\
\mathbf{0} & \sigma_{2}^{2} \mathbf{I}_{N_{2}-n_{2}}
\end{array}\right),
\end{aligned}
$$

where $\mathbf{z}=\left(\mathbf{z}_{s, 1}^{\prime}, \mathbf{z}_{s, 2}^{\prime}, \mathbf{z}_{u, 1}^{\prime}, \mathbf{z}_{u, 2}^{\prime}\right)^{\prime}$. Suppose that now we want to predict the total, $\mathbf{B}=\mathbf{b}=$ $\mathbf{1}_{N_{1}+N_{2}}$. Then, from (13) we obtain the predictor,

$$
\lambda^{\prime} \mathbf{z}_{s}=N_{1} \bar{z}_{s, 1}+N_{2} \bar{z}_{s, 2}
$$

From (15),

$$
\operatorname{MSPE}=\left(N_{1}^{2} \sigma_{1}^{2} / n_{1}\right)\left(1-f_{1}\right)+\left(N_{2}^{2} \sigma_{2}^{2} / n_{2}\right)\left(1-f_{2}\right),
$$

where $f_{1}=\left(n_{1} / N_{1}\right)$ and $f_{2}=\left(n_{2} / N_{2}\right)$. Of course, Eq. 19 is the same estimator of the mean that is used in SRS (e.g., Thompson 1992, p. 103) and Eq. 20 is the variance of the mean estimator used in SRS (e.g., Thompson, p. 103).

Equations 17 and 20 demonstrate that Eq. 15 is a version of block kriging that provides a reduction in variance when sampling finite populations due to the finite population correction factors $f$. In summary, the second-order stationarity assumption for FPBK is a stronger assumption than SRS, and we get the same estimator when assuming independence. However, data often appear spatially patterned, and we can get much more when they are modeled as spatially autocorrelated.

\subsection{Modeling autocorrelation}

To make full use of model-based assumptions, we will need to estimate $\boldsymbol{\Sigma}$ by modeling the spatial autocorrelation in the data. We need to estimate each of the $(i, j)$ entries in $\boldsymbol{\Sigma}$. One such model for spatial covariance (2) is the exponential model,

$$
C(\mathbf{h} \mid \boldsymbol{\theta})= \begin{cases}\theta_{1}+\theta_{2} & \mathbf{h}=0, \\ \theta_{2} \exp \left(-\|\mathbf{h}\| / \theta_{3}\right) & \mathbf{h} \neq 0,\end{cases}
$$

where $\mathbf{h}=\mathbf{s}_{j}-\mathbf{s}_{i}$. There are many others (see Cressie 1993, p. 61). Choosing a model is often a difficult task; one can try several and use crossvalidation (Cressie 1993, p. 101) or AIC (Cressie 1993, p. 104), or make a choice based on experience or a flexible class (at the expense of an extra parameter, Stein 1999, p. 176). It is possible to estimate the parameters of $C(\mathbf{h} \mid \boldsymbol{\theta})$ using method of moments for variograms or covariances (see Cressie 1993, p. 69) and then weighted least squares (see Cressie 1993, p. 99), or by using restricted maximum likelihood (REML, Patterson and Thompson 1971, 1974); see Cressie (1993, p. 92) for spatial 


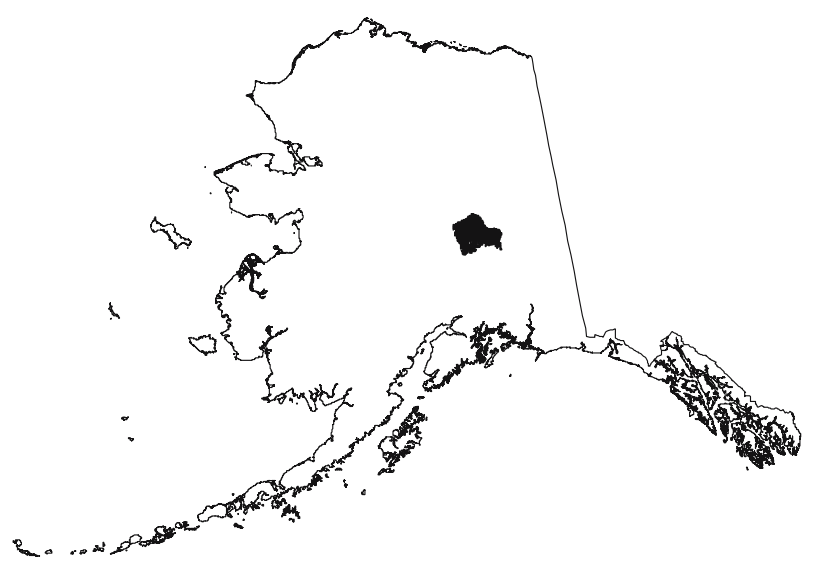

Fig. 1 Map of Alaska, GMU 20A

REML. In the example below, I will use REML. That is, the covariance matrix $\boldsymbol{\Sigma}_{s s}$ depends on the parameters $\boldsymbol{\theta}$ in (21), so minimize

$$
L(\boldsymbol{\theta})=(n-p) \log (2 \pi)+\log \left|\boldsymbol{\Sigma}_{s s}\right|+(\mathbf{z}-\mathbf{X} \widehat{\boldsymbol{\beta}})^{\prime} \boldsymbol{\Sigma}_{s s}^{-1}(\mathbf{z}-\mathbf{X} \widehat{\boldsymbol{\beta}})+\log \left|\mathbf{X}^{\prime} \Sigma_{s s}^{-1} \mathbf{X}\right|,
$$

where $\widehat{\boldsymbol{\beta}}=\left(\mathbf{X}^{\prime} \Sigma_{s s}^{-1} \mathbf{X}\right)^{-1} \mathbf{X}^{\prime} \Sigma_{s s}^{-1} \mathbf{z}$. As a visual diagnostic, I compute the empirical semivariogram,

$$
\hat{\gamma}(\mathbf{h})=\frac{1}{2|N(\mathbf{h})|} \sum_{N(\mathbf{h})}\left[z\left(\mathbf{s}_{i}\right)-z\left(\mathbf{s}_{j}\right)\right]^{2},
$$

where $\mathbf{h}=\mathbf{s}_{j}-\mathbf{s}_{i}, N(\mathbf{h})=\left\{\left(\mathbf{s}_{i}, \mathbf{s}_{j}\right): \mathbf{s}_{j}-\mathbf{s}_{i}=\mathbf{h}\right\}$ and $|N(\mathbf{h})|$ is the number of distinct elements in $N(\mathbf{h})$. The fitted model covariance (21) is readily converted to a semivariogram using the relationship, $\gamma(\mathbf{h} \mid \boldsymbol{\theta})=C(\mathbf{0} \mid \boldsymbol{\theta})-C(\mathbf{h} \mid \boldsymbol{\theta})$.

\section{Examples using moose survey data}

I give an example from a moose survey conducted in Alaska in the fall of 1999. The survey area was game management unit (GMU) 20A, shown as the darkened area within the state of Alaska in Fig. 1. The survey was flown in November after there was sufficient snow cover to allow moose to be readily observed. Moose surveys involve five basic elements that include, (1) defining the survey area, (2) stratifying the area, (3) selecting a sample, (4) surveying the sample of units within the area, and (5) analyzing the data. Within a survey area, sample units are laid out in a grid. The north-south boundaries are based on even increments of latitude ( $2 \mathrm{~min}$, starting at 0 ) and the east-west boundaries are based on increments of longitude (5 min, starting at 0 ). At around 64 latitude, sample unit size is approximately $15 \mathrm{~km}^{2}$. The total area of the survey was $14,878 \mathrm{~km}^{2}$. Figure 2 shows an enlarged view of the $20 \mathrm{~A}$ survey area. The area was stratified into two strata: relatively low moose density (shown as white in Fig. 2) and relatively high moose density (shown by gray in Fig. 2), based on the biologist's knowledge of habitats, moose, and their distribution in previous years. The low stratum consisted of 338 sample units and the high stratum consisted of 649 sample units.

After the area was stratified, a random sample of 86 samples was drawn with 52 from the high stratum and 34 from the low stratum. The sample units were flown and all moose 


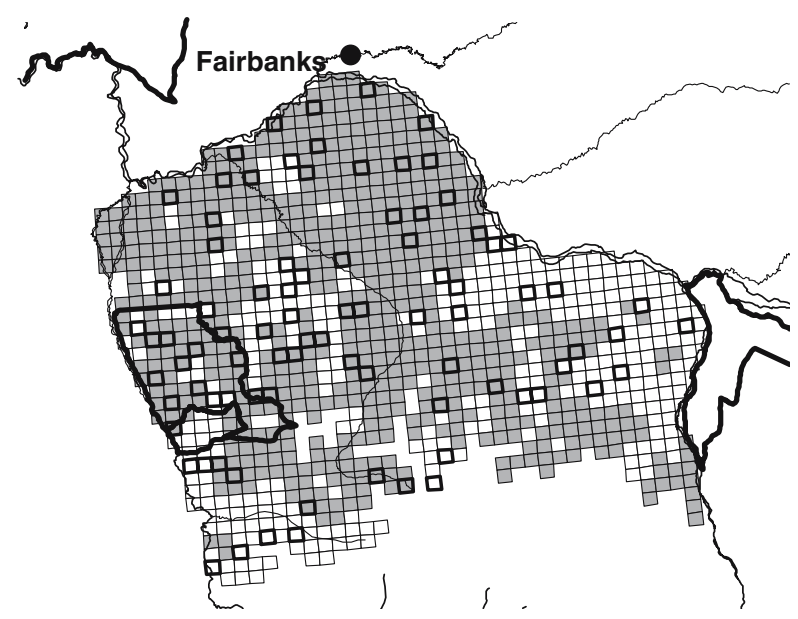

Fig. 2 GMU 20A stratification and samples. The low stratum is shaded white, and the high stratum is shaded gray. Sampled units have a heavy border, and the FTMA is outlined with a heavy border to the left

were counted from the air within each sample. The sampled units are shown with a heavy border in Fig. 2. All counts were first changed to density by dividing the counts by the area of each sample, which varied slightly due to the narrowing of longitude as one moves north. An average of 0.973 moose per square kilometer was counted in the high stratum, and an average of 0.398 moose per square kilometer was counted in the low stratum.

The covariance between sample units was estimated using an exponential model (21) and REML (22). The distance between sample units was computed in kilometers from the center of one sample unit to the center of another. For the high stratum, the estimated parameters in (21) were $\theta_{1}=2.614, \theta_{2}=0.670$, and $\theta_{3}=23.26$. The empirical semivariogram (23) and the fitted model (21) for the high stratum are given in Fig. 3. In Fig. 3, the size of the circle indicates the number of pairs of locations used for each distance class in the empirical semivariogram, and the line is the fitted model. For the low stratum, the estimated parameters in (21) were $\theta_{1}=0.000, \theta_{2}=2.102$, and $\theta_{3}=15.99$. The empirical semivariogram (23) and the fitted model (21) for the low stratum are given in Fig. 4. All covariances between the two strata are assumed to be 0 .

The fitted models, given above, are used to fill in the $(i, j)$ entries in $\Sigma$, including $\boldsymbol{\Sigma}_{u u}$ and $\Sigma_{s u}$. That is, because we assume stationarity, we use the same spatial model for unsampled locations as for sampled locations. Using the estimated $\boldsymbol{\Sigma}$ and $\mathbf{B}=\mathbf{b}=(1 / N)(1,1, \ldots, 1)^{\prime}$ and $\mathbf{X}$ as in (18), the predictor FPBK (13) of the average moose density per sample was estimated to be 0.7613 moose per square kilometer, or a total of 11,327 moose in the whole study area. The estimated standard error of the average moose density using (15) was 0.0043 , yielding an estimated standard error of 978 for the total number of moose. For comparison, using SRS (e.g., Thompson 1992, p. 103), the estimate of total moose abundance was 11,535 with an estimated standard error of 985 . It is generally true that if there is autocorrelation in the data, prediction will be more precise when it uses information about the autocorrelation; thus FPBK has slightly smaller variance than SRS (see Ver Hoef 2002, for more examples and simulations).

Using the same fitted variogram model, it is possible to make a prediction for any subset of samples; i.e., small area estimation. Figure 2 also shows the Ferry Trail Management Area (FTMA) outlined in bold on the left. FPBK yielded an estimate of 1,437 moose in the 


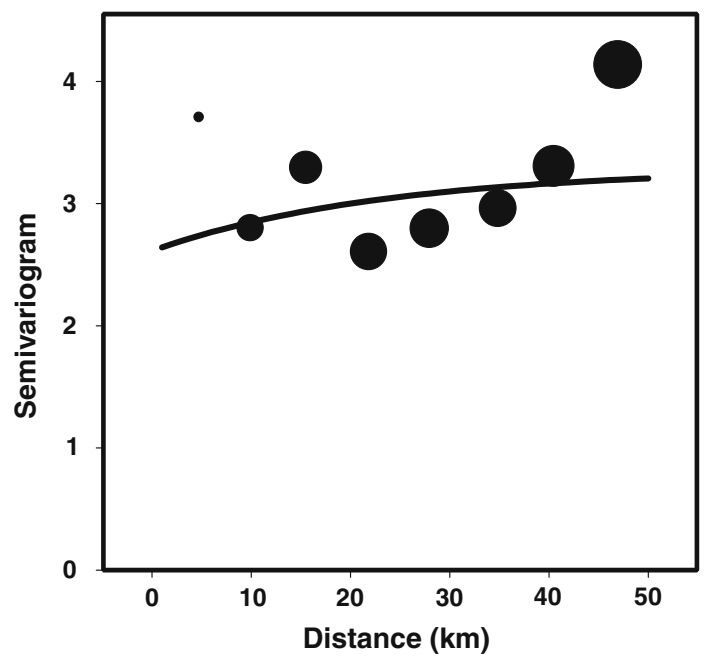

Fig. 3 Empirical (solid circles) and fitted (solid line) semivariogram for high stratum. The size of the circles are proportional to the number of sample pairs for each lag class

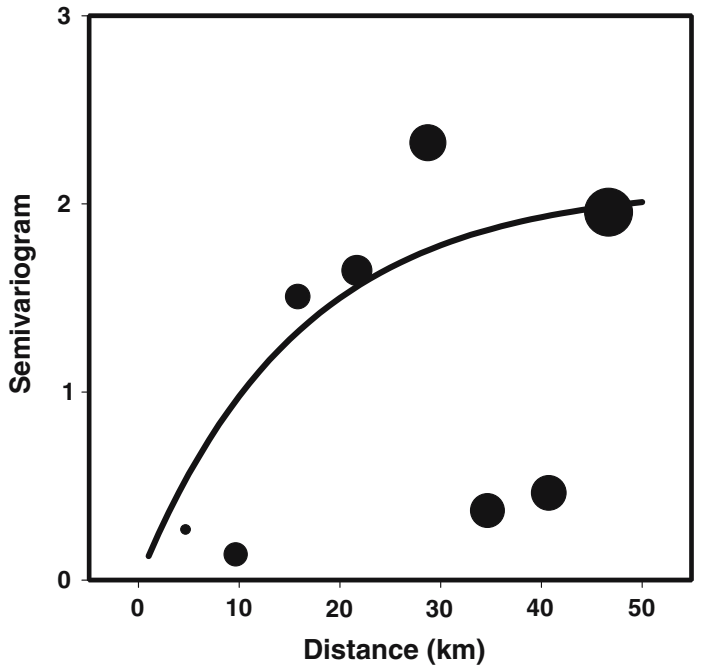

Fig. 4 Empirical (solid circles) and fitted (solid line) semivariogram for low stratum. The size of the circles are proportional to the number of sample pairs for each lag class

FTMA with a standard error of 153. The SRS estimate using only the samples within the FTMA subset (13 highs and 4 lows) yielded an estimated 1,535 moose with an estimated standard error of 227. For small area estimation, the estimated standard error of FPBK was significantly smaller than that of SRS. 


\section{Discussion and conclusions}

Based on over 100 different moose surveys since 1998, and information from simulations in Ver Hoef (2002), the application of geostatistical ideas to finite population sampling gives three main advantages over classical sampling: (1) FPBK is usually more precise than SRS, (2) FPBK allows small area estimation, and (3) FPBK allows nonrandom sampling designs, giving biologists greater flexibility. The increased precision should come as no surprise because FPBK borrows strength from neighboring locations, especially in the case of small area estimation; that is, it uses information outside of the "small area," which is not the case for SRS.

The use of nonrandom designs requires more explanation. Of course, if we had prior knowledge of regions with high values, and only sampled those regions, then we wouldn't do a good job of estimating regions with low values, and hence our overall estimate would not be very good. On the other hand, the model basis of FPBK does not rely on the space of all possible randomizations, but rather the second-order stationarity assumption, and we can use this to our advantage. Systematic designs, as shown by Ver Hoef (2002) and references therein, often have better precision than SRS designs, and are often easier to implement. Systematic designs are "space-filling," and known to be optimal for kriging (Cressie 1993, p. 318). Recall that for kriging, the variance increases most dramatically at the edges, or in regions without samples ("holes"). FPBK operates in the same way, by predicting unsampled units. Hence, it is important to fill gaps and holes. I have used randomized designs, but noticed that often, by chance, there were holes and gaps at edges, and so I allocated further samples by placing them, subjectively, to fill holes and gaps. As a final note on flexibility, sampling often has several goals, including estimating an overall total as well as a total from a smaller area. Depending on the importance of the small area, additional samples can be allocated to it, or near it, which will give a more precise estimate of the small area at the expense of the overall area. There is much work to be done on the whole issue of optimal designs, but it is beyond the scope of this paper. The few guidelines given above, however, can help increase precision through more flexible designs. Note that others are developing "space-filling" designs, like systematic designs, that do have random properties (e.g., Stevens and Olsen 2004). Such designs can also be used with FPBK, but with the modelbased assumption of stationarity.

FPBK also has an advantage over block kriging because FPBK incorporates a finite population correction factor that reduces the prediction variance. In the example above, approximately $9 \%$ of the population was sampled, and it often gets as high as $30 \%$ for moose surveys in Alaska. I have also used FPBK for simulated examples and data other than moose surveys (Ver Hoef 2002), and obtained similar results and conclusions.

SRS allows a separate variance for each stratum. The analogy for a model-based approach is to have a separate spatial process for each stratum. Geostatistical methods assume a constant variance for a spatial process, and stratification helps meet that assumption. When considering multiple spatial processes induced by stratification, it is possible (even desirable) to model cross-correlation between strata processes. I have investigated this for moose surveys in Alaska and found little or no cross-correlation so that it did not affect the predictions or standard errors. An alternative to stratification is transforming the data to stabilize variance. This is useful when continuous covariates (e.g., elevation) are used in the design matrix of the linear model. These methods require an unbiased backtransformation (similar to transgaussian kriging, Cressie 1993, p. 137), and results will be presented later. 
Acknowledgements Financial support for this work was provided by Federal Aid in Wildlife Restoration to the Alaska Department of Fish and Game.

\section{References}

Bolfarine H, Zacks S (1992) Prediction theory for finite populations. Springer-Verlag, New York, p 207

Cressie N (1993) Statistics for spatial data, Revised edn. John Wiley and Sons, New York, p 900

Fay R, Harriot R (1979) Estimates of income for small places: an application of James-Stein procedures to census data. J Am Stat Assoc 74:269-277

Gasaway WC, DuBois SD, Reed DJ, Harbo SJ (1986) Estimating moose population parameters from aerial surveys. Biological Papers of the University of Alaska, vol 22, p108

Ghosh M, Meeden G (1986) Empirical Bayes estimation in finite population sampling. J Am Stat Assoc 81:1058-1062

Patterson HD, Thompson R (1971) Recovery of interblock information when block sizes are unequal. Biometrika 58:545-554

Patterson HD, Thompson R (1974) Maximum likelihood estimation of components of variance. In: Proceedings of the 8th International Biometric Conference, Biometric Society, Washington, DC, pp 197-207

Prasad NGN, Rao JNK (1990) On the estimation of mean square error of small area predictors. J Am Stat Assoc 85:163-171

Stein ML (1999) Interpolation of spatial data: some theory for Kriging. Springer, New York, p 247

Stevens DL Jr , Olsen AR (2004) Spatially-balanced sampling of natural resources. J Am Stat Assoc 99:262278

Thompson S (1992) Sampling. John Wiley and Sons, New York, p 343

Ver Hoef JM (2002) Sampling and geostatistics for spatial data. Ecoscience 9:152-161

\section{Author biography}

Jay M. Ver Hoef completed most of this work as a biometrician for the Wildlife Conservation Division of the Alaska Department of Fish and Game. He is currently a statistician with the National Marine Mammal Laboratory in Seattle, where he serves as a statistical consultant and continues his statistical research interests. $\mathrm{He}$ is also an adjunct professor of statistics with the Mathematics Department of the University of Alaska, Fairbanks, and a fellow of the American Statistical Association. He received his B.S. in botany from Colorado State University, Fort Collins, his M.S. in botany from the University of Alaska, Fairbanks, and his Ph.D., a co-major in statistics and EEB (ecology and evolutionary biology), from Iowa State University, Ames, Iowa. 